\title{
Development and application of the modified strong dipole-proton coupling model for KDP-type crystals
}

\author{
DALIBOR MERUNKA and BORIS RAKVIN \\ Ruđer Bošković Institute, P.O.Box 180, 10002 Zagreb, Croatia
}

PACS index: 77.84.Fa; 77.80.Bh; 76.30.Rn; 76.60.Cq; 76.60.Gv

Keywords: KDP-type ferroelectrics; isotopic effect; order-disorder phase transition; displacive phase transition; paramagnetic probe; KDP-type antiferroelectrics

\begin{abstract}
The modified strong dipole-proton coupling (MSDPC) model, which predicted several static and dynamic dielectric properties of KDP-type ferroelectrics, was used to investigate local properties of these crystals. The results showed both order-disorder and displacive characteristics of pure KDP and DKDP, as in many other experimental studies (NMR, neutron scattering) of local properties. Paramagnetic center $\mathrm{SeO}_{4}{ }^{3-}$ introduced in KDP and DKDP lattice as a local probe in EPR technique was treated within MSDPC model as a "soft" impurity. Simulated dipole reorientational dynamics of the impurity showed characteristics similar to the slow local dynamics of the probe detected in an EPR experiment. Additionally, the MSDPC model was adapted to describe dielectric properties of KDP-type antiferroelectrics. Antiferroelectric phase transition of the first order and isotopic shift in $T_{c}$ were reproduced.
\end{abstract}




\section{INTRODUCTION}

The origin of a large isotopic effect on $T_{c}$ when $\mathrm{H} \rightarrow \mathrm{D}$ and a phase transition mechanism for ferroelectrics of $\mathrm{KH}_{2} \mathrm{PO}_{4}$ or KDP-type are still unclear. The proton tunneling model, the first model that explained the isotopic effect, predicted the displacive mechanism [1]. However, more recent lattice dynamics measurements have indicated the order-disorder mechanism. According to the strong dipole-proton coupling (SDPC) model, a difference in ground-state energy between $\mathrm{H}$ and $\mathrm{D}$ causes the isotopic effect [2]. This model was modified (MSDPC model) by inclusion of transversal (perpendicular to the ferroelectric $c$-axis) dipole components, which reproduced better value of $T_{c}$ for $\mathrm{KD}_{2} \mathrm{PO}_{4}(\mathrm{DKDP})$ and the transversal dielectric constant anomaly [3]. The molecular dynamics (MD) simulations performed to study longitudinal ("soft mode") and transversal polarization dynamics in KDP and DKDP yielded the results similar to experimental data [4]. In this study local static and dynamic properties of these systems were investigated by applying MSDPC model. The obtained results were compared with experimental data from NMR, EPR, and neutron scattering measurements. In addition, MSDPC model was adapted to describe antiferroelectrics of KDP-type i.e. $\mathrm{NH}_{4} \mathrm{H}_{2} \mathrm{PO}_{4}(\mathrm{ADP})$ and $\mathrm{ND}_{4} \mathrm{D}_{2} \mathrm{PO}_{4}$ (DADP).

\section{LOCAL STATIC PROPERTIES}

MD simulations and model parameters defined earlier [2-4] were used to investigate the local properties of MSDPC model. The induction of $\mathrm{PO}_{4}$ dipole is related to the shift of $\mathrm{P}$ atom and mean-square deviation from the mean value of the $c$-component of $\mathrm{PO}_{4}$ dipole

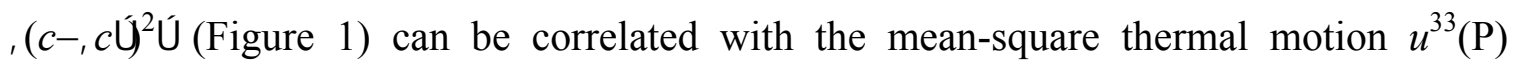
obtained in the neutron scattering experiment [5]. The calculated temperature dependence 
of the mean-square of the $c$-component of $\mathrm{PO}_{4}$ dipole,$c^{2}$ Úcan be approximated with ,$c^{2} \dot{\ddagger} \Delta+\gamma T$ well above $T_{c}$ and the fast decrease of , $\left(c-, c \cup^{2}\right.$ Úbelow $T_{c}$ occurs (Figure 1). This behavior supports existence of the temperature-independent constant $\Delta$, which has larger value for DKDP than for KDP and disappears in the ferroelectric phase in accordance with the order-disorder type of phase transition. Similar temperature behavior and isotopic effect in $\Delta$ shows $u^{33}(\mathrm{P})$ [5]. The value of , $c^{2}$ Úshows anomalous increase by lowering temperature towards $T_{c}$ and continues to increase below $T_{c}$. Experimental parameters that can be related to,$c^{2}$ Ú such as ${ }^{31} \mathrm{P}$ isotropic chemical shift [6] and ${ }^{75} \mathrm{As}$ quadrupole coupling constant in $\mathrm{KH}_{2} \mathrm{AsO}_{4}$ [1], show similar temperature behavior. These experimental data indicate a displacive component in the phase transition mechanism [6].

\section{PARAMAGNETIC PROBE}

The $\mathrm{PO}_{4}{ }^{3-}$ group of the KDP lattice can be replaced with the paramagnetic probe $\mathrm{SeO}_{4}{ }^{3-}$ to study local lattice dynamics by EPR spectroscopy. The probe exhibits a local lowfrequency polarization fluctuation mode at EPR time scale $\left(10^{7}-10^{10} \mathrm{~Hz}\right)$ in the paraelectric phase. The obtained motional dynamics was usually described by simple Arrhenius-type correlation time $\tau=\tau_{0} \exp (\Delta E / k T)$ [7]. In the MSDPC model, the $\mathrm{SeO}_{4}{ }^{3-}$ probe is described as "soft" impurity with $A_{\text {probe }}$ value of the elastic constant for the dipole $c$-component induction lower than the $A$ value for the original $\mathrm{PO}_{4}{ }^{3-}$ group. For $A_{\text {probe }}=0.45 A$, the MD simulations in the paraelectric phase of KDP and DKDP show the effective local double-well potential of the probe and the temperature dependence of calculated correlation times $\tau$ for the dipole reorientational jumps over the barrier can be fitted to the Arrhenius law (Figure 2). The fitted parameters for KDP are close to the 
experimental ones, and the experimentally detected higher activation energy $(\Delta E)$ in DKDP is also reproduced [7].

\section{ANTIFERROELECTRICS OF KDP-TYPE}

By replacing $\mathrm{K}$ ions in the KDP lattice with $\mathrm{NH}_{4}$ group, the obtained crystal lattice exhibits antiferroelectric (AFE) phase transition of the first order and the isotope effect in $T_{c}$ [8]. The ordered phase is characterized by the four types of AFE domains having transversal unit dipoles. The effect of the $\mathrm{NH}_{4}$ group in ADP is introduced in the MSDPC model by additional force, which is induced by the transversal $\mathrm{PO}_{4}$ dipole and acts on the next nearest $\mathrm{H}(\mathrm{D})$. The force is proportional to the dipole with the constant $L_{\perp}$ and its orientation is chosen so that dipole orientation at $T=0 \mathrm{~K}$ corresponds to one of the AFE domains. Due to similarity of hydrogen bond lengths, the parameters $h$ and $I$ have the same value for ADP (DADP) as for KDP (DKDP) [2]. To reproduce experimental values of dielectric constants $\varepsilon_{a}$ and $\varepsilon_{c}$ along the $a$ and $c$-axis, the values of the previously defined parameters for KDP (DKDP) [3] are adjusted for ADP (DADP), as follows: $A=2.474 h^{\mathrm{H}} /\left(\mu_{s}^{\mathrm{H}}\right)^{2}, A_{\perp}=0.174 h^{\mathrm{H}} /\left(\mu_{s}^{\mathrm{H}}\right)^{2}, I K=0.847 h^{\mathrm{H}} / \mu_{s}^{\mathrm{H}}, I K_{\perp}=0.226 h^{\mathrm{H}} / \mu_{s}^{\mathrm{H}}$, with the new parameter value $I L_{\perp}=0.0023 h^{\mathrm{H}} / \mu_{s}{ }^{\mathrm{H}}$. Calculated results for $\varepsilon_{a}$ and $\varepsilon_{c}$ in ADP and DADP show sharp discontinuity at $T_{c}$ as expected for the first order transition, as well as isotopic shift in $T_{c}$ (Figure 3). 


\section{CONCLUSION}

Several dielectric and local properties as well as isotope effects for KDP can be predicted and fairly good described with MSDPC model without including proton tunneling. This

model can also predict the first order AFE phase transition in ADP by including an additional interaction between dipole and the second nearest protons. 


\section{REFERENCES}

1. R. Blinc and B. Žekš, Ferroelectrics 72, 193 (1987).

2. H. Sugimoto and S. Ikeda, Phys. Rev. Lett. 67, 1306 (1991).

[www http://link.aps.org/abstract/PRL/v67/p1306]

3. D. Merunka and B. Rakvin, Phys. Rev B 61, 11967 (2000).

[www http://link.aps.org/abstract/PRB/v61/p11967]

4. D. Merunka and B. Rakvin, Phys. Rev B 66, 174101 (2002).

[www http://link.aps.org/abstract/PRB/v66/e174101]

5. R. J. Nelmes, Ferroelectrics 71, 87 (1987).

6. A. Bussmann-Holder, N. Dalal, R. Fu and R. Migoni, J. Phys.: Condens. Matter 13,

L231 (2001). [www http://stacks.iop.org/0953-8984/13/L231]

7. B. Rakvin and N. Dalal, Phys. Rev. B 41, 608 (1990).

[www http://link.aps.org/abstract/PRB/v41/p608]

8. S. Havlin, Ferroelectrics 71, 183 (1987). 


\section{FIGURE CAPTIONS}

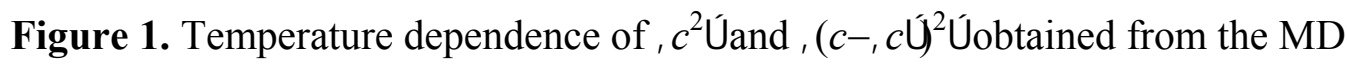
simulation on KDP and DKDP system of $256 \mathrm{PO}_{4}$ dipoles, where $c$ is value of $\mathrm{PO}_{4}$ dipole component along $c$-axis relative to the saturated value $\mu_{s}{ }^{\mathrm{H}}=4.8 \times 10^{-30} \mathrm{C} \mathrm{m}$ for KDP.

Figure 2. The MD simulation results for inverse reorientational correlation time of the "soft" impurity with $A_{\text {probe }}=0.45 A$ embedded in KDP and DKDP system of $107 \mathrm{PO}_{4}$ dipoles in paraelectric phase. Lines denote the best fits to the Arhhenius law.

Figure 3. Results obtained from the MD simulation for (a) $\varepsilon_{a}$ and (b) $\varepsilon_{c}$ on the ADP and DADP system of $108 \mathrm{PO}_{4}$ dipoles. Lines denote experimental values [8]. 
D. Merunka and B. Rakvin, Figure 1.

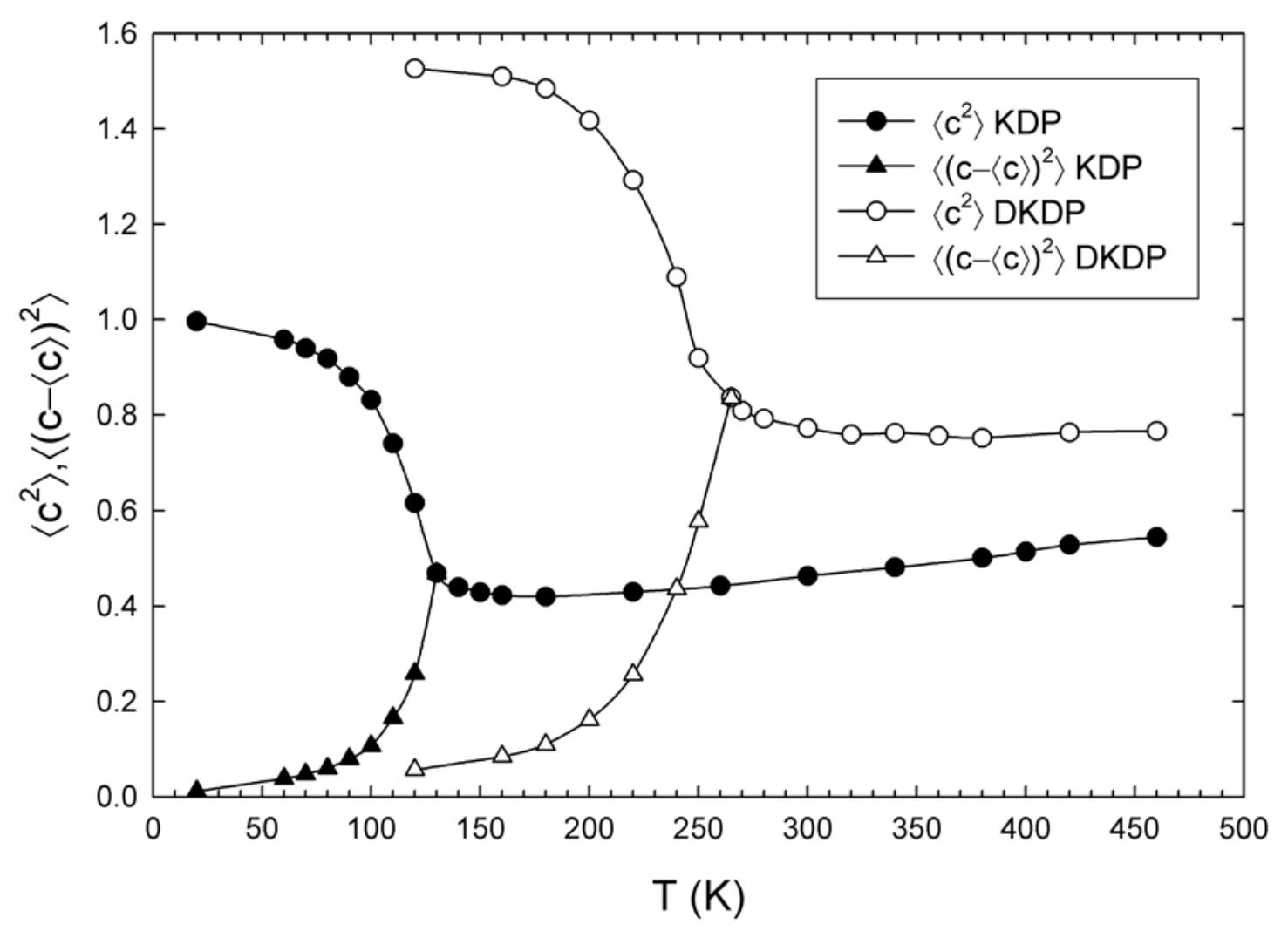


D. Merunka and B. Rakvin, Figure 2.

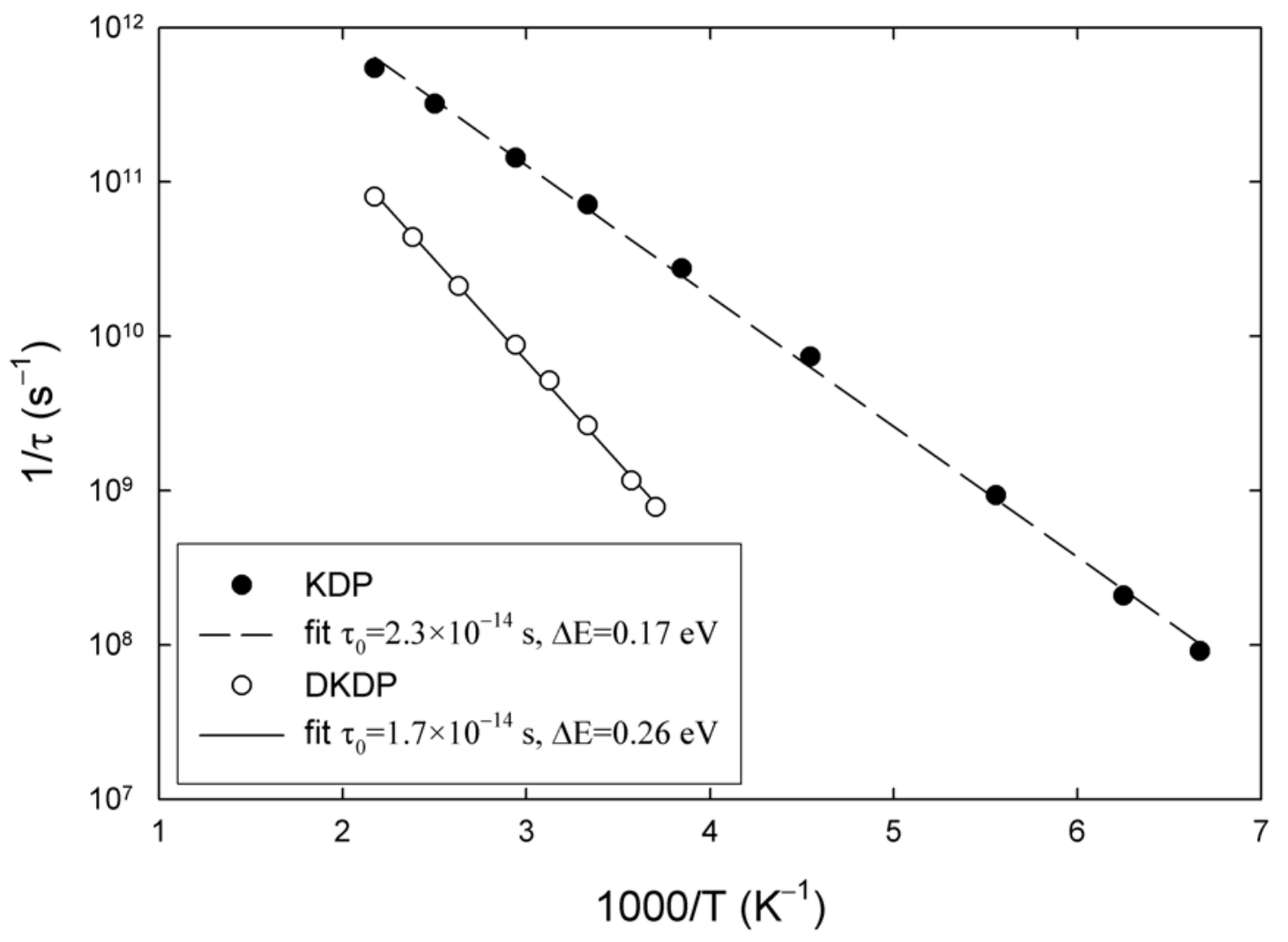


D. Merunka and B. Rakvin, Figure 3.
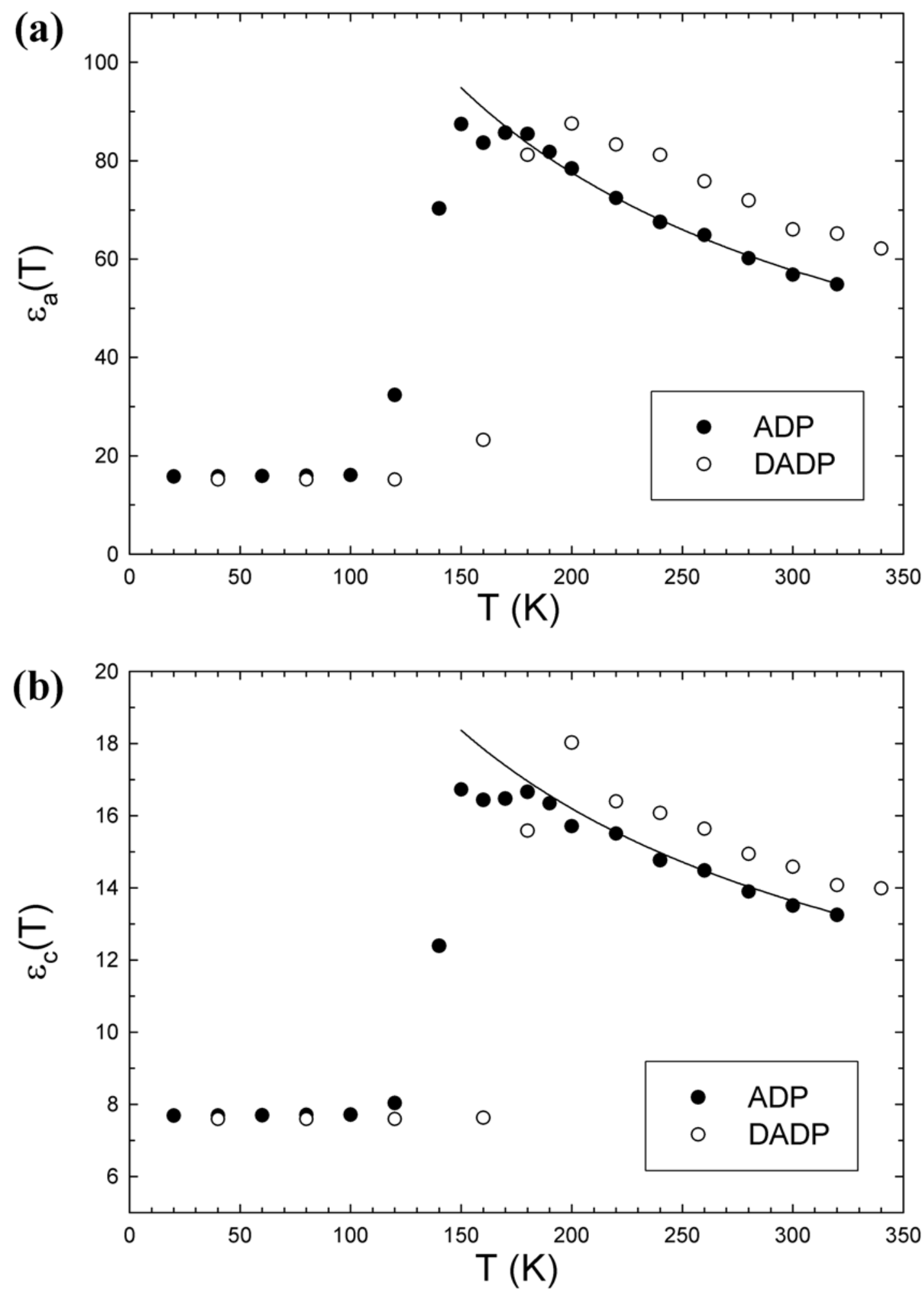